\title{
Arrhythmia Detection using Pan-Tompkins Algorithm and Hilbert Transform with Real-Time ECG Signals
}

\author{
${ }^{*}$ Deniz Balta and Esra Melike Akyemiş \\ ${ }^{* 1}$ Faculty of Computer and Information Science, Department of Software Engineering Sakarya University, Turkey
}

\begin{abstract}
Electrocardiography (ECG) is a form of examination based on printing on millimetric paper the contraction and relaxation phases of the atria and ventricles of the heart, the electrical activity that occurs during the stimulation of the heart and the transmission of the stimulus. For the first diagnosis of diseases such as heart attacks, it is very important to make this preliminary impression and inform the doctor and patient as soon as possible.In this study, any arrhythmia risk can be determined by examining the heart rate and QRS width values calculated by graphing the signal value coming from the electrodes of the Electrocardiography monitor taken for heart rhythm monitoring in hospitals and by detecting the QRS points from the signal data with the Pan Tompkins algorithm and Hilbert Transform method. The image of the ECG graph and the signal analysis results, which are the result of the graphing of the data, can be sent via e-mail. In line with the values obtained in this study, it has been determined that the Pan Tompkins algorithm's signal detection accuracy, sensitivity and accurate prediction ratio gives better results than Hilbert Transform method during the detection of peaks from ECG signals.
\end{abstract}

Key words: Pan Tompkins, Hilbert Transform, Electrocardiography, AD8232 ECG Sensor

\section{Introduction}

According to the report of the World Health Organization (WHO), cardiovascular diseases (CVD) are considered as one of the leading causes of death globally, taking an estimated 18 million lives each year. This number is approximately $32 \%$ of the world's population [1]. Irregular heartbeats sometimes show no symptoms until they become serious, and patients experience palpitations and chest pain. While several types of arrhythmias are not serious, they are a precursor to certain diseases such as stroke, heart failure, and cardiac arrest.

Electrocardiography has been used as a diagnostic tool for heart diseases for many years. The electrocardiogram (ECG) reflects the electrical polarization and depolarization of the heart chambers. In addition, the ECG, which is used for the diagnosis of cardiovascular diseases, is a device that provides graphical recording of the electrical activity that occurs during the contraction of the heart. Thanks to the ECG, which has become the gold standard in the evaluation of heart function, contraction of the heart muscle, rhythm and conduction disorders can be understood [2, $3]$. The procedure, which does not require the patient to make any special preparations, is performed in a special room. Electrodes connected to the ECG device are attached to the chest, legs and arms. During 2-3 minutes of shooting, the patient is asked not to move or talk too much. After the shooting is completed, the data obtained by the ECG device while the person is being prepared is converted into a graphic and printed on a paper called an electrocardiogram. The physician evaluates the ECG result together with the examination findings and heart diseases can be determined. Heart conditions are detected by arrhythmia (irregular heartbeat) in the ECG

*Corresponding author: Address: Faculty of Computer and Information Science, Department of Software Engineering Sakarya University, 54187, Sakarya TURKEY. E-mail address: ddural@ sakarya.edu.tr, Phone: +902642955895 
signals.

However, it will be faster to analyze the ECG data with a machine or a more accurate term, software instead of a physician. And with this speed, it will enable the possibility of heart risk and heart rhythm disorders to be diagnosed earlier, which is noticed much later, often late in their treatment.

There are many studies in the literature for the detection of arrhythmia. Generally, studies are divided into two parts. The first part is the detection of QRS points by taking ECG data and feature extraction, the second part is the classification part where the values obtained as a result of the first stage are analyzed by various artificial intelligence algorithms. In the feature extraction phase, it has been seen that many methods such as Wavelet Transform, Hilbert Transform (HT), PanTompkins algorithm (PT) are frequently used for the determination of R peaks in the literature. For classification, various classifiers such as k-nearest neighbors $(\mathrm{kNN})$, neural networks and support vector machine (SVM) are used.

In a study conducted for the diagnosis of arrhythmia in the literature, using the Pan Tompkins algorithm and the MIT-BIH database, the peak R point of the ECG data is found by the Pan Tompkins algorithm, 5 features are extracted from this value, and arrhythmia is detected in artificial neural networks together with the data set [4]. In another study, a graphical user interface (GUI) was developed for arrhythmia detection using Pan-Tompkins on electrocardiogram signal data obtained from the MIT-BIH arrhythmia database. Various parameters were calculated in the PanTompkins algorithm and modifications were made to the algorithm to detect cardiac abnormalities [5]. In another study in which MIT-BIH arrhythmia database ECG recordings were determined using R peaks, Hilbert Transform and Pan-Tompkins algorithms, it was said that the Pan-Tompkins algorithm gave more accurate and sensitive results [6]. In a study in which the improved Pan Tompkins algorithm was proposed, it was observed that the proposed algorithm successfully detected the QRS complex and significantly reduced the noise in the ECG signal [7]. In another study, data is obtained from the AD8232 sensor with raspberry pi, and its transport by mobile and arrhythmia detections are returned as mobile messages [8]. In another study similar to this, the same method was made with Arduino UNO, the data was obtained and classified by serial communication [9]. Unlike the existing studies, in this study, the ECG signal data obtained from the AD8232 sensor is transmitted with the ESP8266 Wifi module, the collected data are analyzed separately with the Pan Tompkins algorithm and Hilbert Transform method, which are frequently used in studies on this subject, and the methods used are compared. In addition, the results obtained are instantly communicated to the relevant users via e-mail.

\section{Materials and Method}

\subsection{General Structure of Proposed Architecture}

In this study, an architectural proposal for ECG measurement and monitoring systems does not depend on a single measurement environment. An architectural design that provides flexible, scalable, distributed and end-to-end transmission is realized. In addition, different types of sensor 
can be included into the architecture and the number of sensor nodes can be easily increased according to different application areas.

The proposed system consists of three parts that are a sensor network, the servers and the users. The first part of the architecture is considered as a local node, in which measuring devices (AD8232 sensors on ESP8266 via Arduino). The local nodes are used for data gathering and data fusion modules of the proposed ECG monitoring system via measurement devices.

The second part of the proposed system architecture consist of different kind of servers. There are 3 type of servers that are called mail server, database server and application servers in the system. These servers are located on a virtual server. The mail server is responsible for the delivering result mail to the users. The application server retrieves the ECG measurement data that are sent and recorded in the database server over a period of time and determines whether there is a risk of arrhythmia by calculating the heart rate with the detection of QRS points using Pan Tompkins algorithm and Hilbert Transforms method. In addition it makes graphical representations of ECG measurement parameter on the system. Finally, obtained results are delivered to the users who are last part of the system via email, so the users can reach results instantly. The block diagram of the study is shown at the Figure 1.

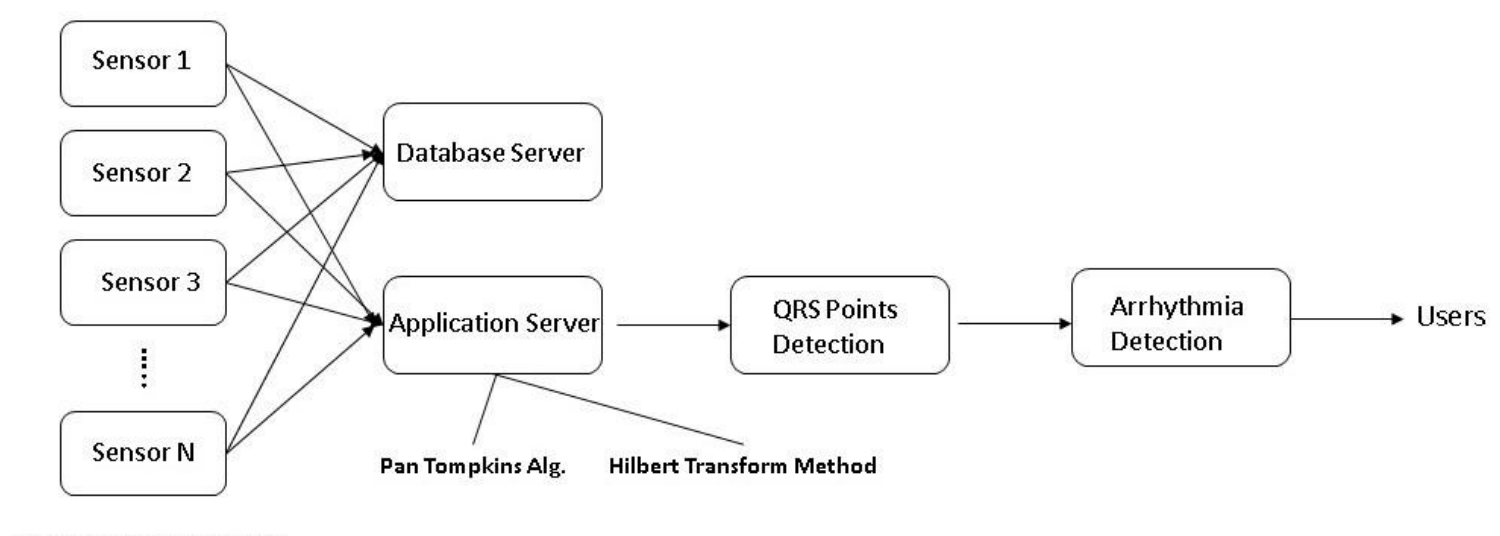

AD8232 ECG Sensors

Figure 1. Block diagram of the study

\subsection{Methods}

\subsubsection{Data Collection}

The first stage of the study is data collection. ECG signals are collected using AD8232 sensor. The ECG data read from the sensor is transferred to the client online via the ESP8266, where it is converted into a graphical display in real time. This image is a variety of graphical representation of sensor measurement data over time. The process of reading and transferring the data from the sensor is carried out with the client-server architecture. The collected sensor data and the resulting 
graphical image are saved in an external file for the determination of the heart rate points to be made later.

In the proposed system, the electrical activity measured with the AD8232 sensor can be plotted as an EKG or electrocardiogram and output as an analog reading. ECGs can contain extremely noisy data, the AD8232 Single-End Heart Rate Monitor acts as an op-amp to help easily obtain a clear signal from the PR and QT intervals. In addition, the AD8232 is an integrated signal conditioning block for ECG and other biopotential measurement applications. It is designed to extract, amplify and filter small biopotential signals in noisy conditions such as movement or remote electrode placement. ESP8266 module on Arduino IDE was used in order to transfer the data coming from AD8232 sensors to the internet environment and to monitor instant measurements in the system. In this way, the system has become flexible, interoperable and accessible. The pins that need to be installed on the ESP8266 to form a monitor with the data received from the AD8232 sensor should be as in Figure 2.

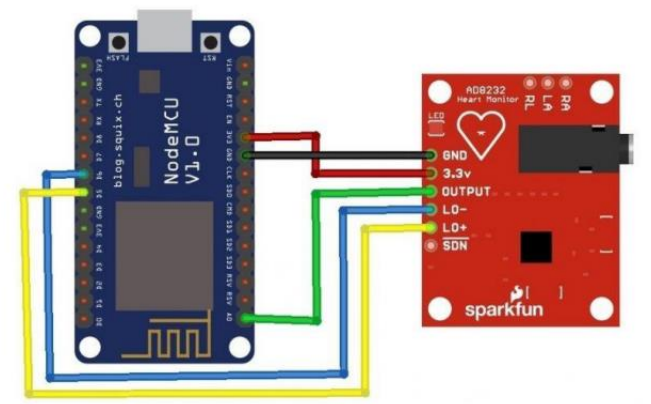

Figure 2. A circuit digram for Interfacing AD8232 ECG Sensor with NodeMCU ESP8266 [10]

The closer the sensor pads are attached to the heart during ECG measurement, the more accurate the measurement will be. That's why the cables are color coded to help determine the correct placement. And the body placement of these colors is shown in Figure 3. Accordingly, pads should be placed so that the red wire is either on the right arm or right chest, the yellow wire is on the left arm or left chest, and the green wire is on the right leg or right groin.



Figure 3. Typical sensor replacement [11] 


\subsubsection{QRS Points Detection}

The QRS complex is formed by a downward deflection (Q wave), a high upward deflection ( $\mathrm{R}$ wave), and a final downward deflection ( $\mathrm{S}$ wave) as shown in Figure 4. The $\mathrm{P}$ wave represents the atrial depolarization time. Under normal conditions, the average wave duration is between 0.06 $0.08 \mathrm{~s}$. Its repolarization is mixed into the QRS. The time from the beginning of the $\mathrm{P}$ wave to the onset of the QRS is expressed as the P-Q segment and represents the time it takes for the impulse to be delivered to the ventricles.

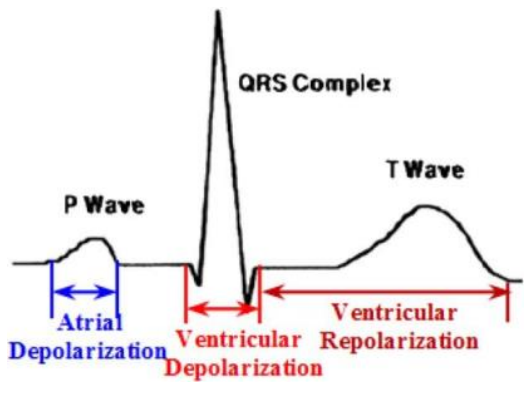

Figure 4. QRS Complex [12]

The QRS Complex gives the sum of the ventricular depolarization and atrial repolarization times. It is the highest positive wave in the EKG. Under normal conditions, it is less than 0.12 seconds on average and has an amplitude of 1-2 $\mathrm{mV}$. Prolongation of the QRS duration indicates that conduction is an abnormal condition in the ventricles. The $\mathrm{T}$ wave indicates the ventricular polarization time and normally lasts $120-160 \mathrm{~ms}[13,14]$.

Basic parameters are important in a normal beating phase of a heart. The shape and duration of the waves, the relations of the $\mathrm{P}$ wave, QRS complex and T wave with each other, and the R-R intervals should be examined in detail. In this regard, the heart rate should be found and evaluated for the detection of abnormality, which is between 70-100 for children and 60-100 for adults. The heart rate can be estimated from the R-R interval value. If the heart rhythm is regular, that is, the sequential RR intervals are fairly constant, it can give a reasonably accurate heart rate reading of $1500 / \mathrm{R}-\mathrm{R} \mathrm{mm}$. Conversely, if the R-R intervals are changing, it is best to specify the number of R$\mathrm{R}$ intervals. How many $\mathrm{R}-\mathrm{R}$ intervals are determined within 10 seconds and the value found is multiplied by 6 . Considering the sensor errors, the irregular method was preferred in this project. In the study, an interval number is calculated for every 10 seconds of a one-minute data value set, and the heart rate is calculated and averaged. That is, the average heart rate per minute is calculated $[13,14]$.

In this study, heart rate $\mathrm{Q}, \mathrm{R}$ and $\mathrm{S}$ points were determined by the Pan-Tompkins algorithm and Hilbert Transform method, which are frequently used in the literature, over the values obtained from the AD8232 sensors during the data collection phase. The obtained results were compared with the normal values. In this way, if there is an irregularity in the heartbeat, that is, if there is an arrhythmia, it can be detected. 


\subsubsection{Pan-Tompkins Algorithm}

The Pan-Tompkins algorithm is an algorithm commonly used to detect QRS points in electrocardiographic signals. With this algorithm, a series of filters are applied to highlight the frequency content of rapid cardiac depolarization and background noise is removed. It then applies adaptive thresholds to detect peaks of the filtered signal. The algorithm proposed by Jiapu Pan and Willis J. Tompkins in 1985 was tested using noisy and noiseless data on MIT/BIH data, an arrhythmia database. It was stated that 99.3 percent of QRS complexes were detected correctly during the performance evaluation phase [15].

The block diagram of the Pan Tompkins algorithm applied to the ECG data in the study is shown in the Figure 5. According to this diagram, a band-pass filter is first applied on the data to increase the low signal-to-noise ratio. In the band-pass filter part, two filters are applied as low-pass and high-pass filters in order to filter the ECG signal from noise. A filter bandwidth of $5-15 \mathrm{~Hz}$ is applied to maximize QRS contribution and reduce noise, baseline drift, power line interference, and P-wave / T-wave frequency content. In the next step, a derivative filter is applied to provide information about the slope of the QRS. The filtered signal is then squared to improve QRS points and reduce the possibility of erroneous recognition of peaks. Next, a moving average filter is applied to provide information about the duration of the QRS complex [16].

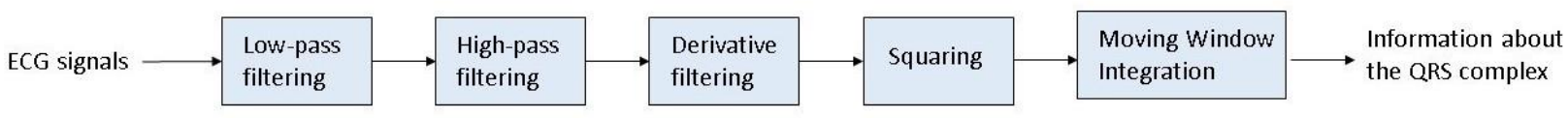

Figure 5. The block diagram of the Pan Tompkins algorithm

\subsubsection{Hilbert Transform}

Hilbert transform is a method that can distinguish between dominant peaks in ECG signals and therefore accurately detects R-peaks. Due to this feature, it is frequently preferred during Arrhythmia detection on ECG data. Various noises such as electrode contact noise that may be present in ECG signals affect frequency resolution, signal quality and greatly affect the structure of the ECG signal, which covers the vital foundations of the heart. The important thing in the ECG signal is to eliminate infected noise and increase accuracy and reliability for better analysis. Although the ECG signal is not inherently stationary, it is possible to visually detect the position of the R-peak by having the robust filtering algorithm combined with the well-known Hilbert Transform to derive the analytical representation of the ECG signal $[6,17]$.

The main benefit of using the Hilbert transform is its single functional feature. This means that when there is a change in the input signal, the Hilbert transformed signal will cross the horizontal axis. This feature is suitable for the detection of QRS complex.

The proposed method is very conveniently described to efficiently detect $\mathrm{R}$ peaks. A real-valued time function $\mathrm{y}(\mathrm{t})$ and the Hilbert transform of the given signal are calculated with the following 
formula 1. Here ' $\mathrm{H}$ ' is the Hilbert transform operator and $\mathrm{y}(\mathrm{t})$ is the Hilbert transform of signal $y(t)$. The Hilbert transform provides a time varying and a linear function of $y(t)$ [17].

$$
H[y(t)]=\frac{1}{\pi} \int_{-\infty}^{\infty} y(\tau) \frac{1}{t-\tau} d \tau
$$

\section{Results and Discussion}

With the application designed in this study, QRS points were determined on the graphical values obtained from AD8232 ECG sensors by using Pan Tompkins and Hilbert Transform methods. The interface of the application is shown in the Figure 6. According to the results obtained when the application was run in the study, it was observed that the Pan Tompkins method was able to detect more QRS compared to the Hilbert Transform method. However, it was observed that the calculated heart rates were the same for both methods. Anomaly status determinations for QRS width give the same result according to both algorithms.

As a result, according to the tests made, it has been seen that both algorithms give good results and can be easily preferred in such studies. However, according to the results obtained from the Pan Tompkins algorithm, it was seen that the signal detection accuracy, sensitivity and accurate prediction ratio performed better than the Hilbert Transform method. In addition, since the Hilbert Transform method is based on a continuous wavelet transform, it has been found to have a very complex computational cost. In practice, unlike the literature, after the ECG data is obtained and the values are analyzed, the necessary message content and ECG graphic image are sent to the users via e-mail. Thus, instantaneous access to the results can be achieved in case of possible heart rhythm disturbance.

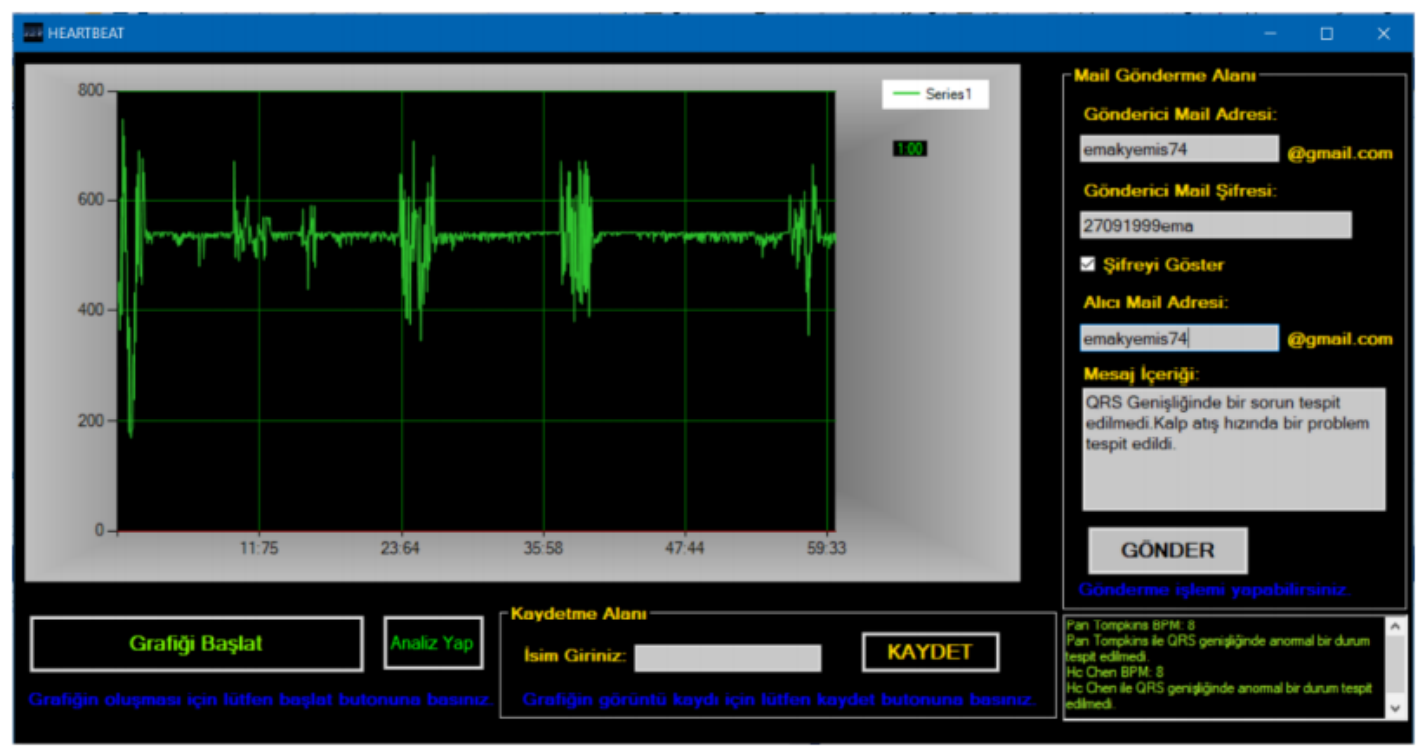

Figure 6. The interface of the application 


\section{Conclusions}

In this study, ECG image data obtained from AD8232 sensors were analyzed using Pan Tompkins algorithm and Hilbert Transform method, and possible heart rhythm disorders were tried to be diagnosed in patients by determining QRS values. Although the number of peaks ( $\mathrm{R}$ point) obtained as a result of the calculated values were different in practice, it was found that both calculated the heart rate obtained from the peak value and evaluated the QRS amplitude found the same. However, the Hilbert Transform method is complex and has a high computational cost, whereas the signal detection accuracy, sensitivity and accurate prediction rate of the Pan Tompkins algorithm are better than the Hilbert Transform method. For this reason, it is clear that the Pan Tompkins algorithm will be a more effective choice in such studies. In future studies, it is aimed to make a more accurate and autonomous disease diagnosis by using various classification algorithms on the data obtained as a result of feature extraction.

\section{References}

[1] World Health Organization. Cardiovascular diseases report 2021. https://www.who.int/newsroom/fact-sheets/detail/cardiovascular-diseases-(cvds). Last accessed date 10.07.2021.

[2] Perlman O, Katz A, Weissman N, Amit G, Zigel Y. Atrial Electrical Activity Detection Using Linear Combination of 12-Lead ECG Signals IEEE Transactions On Biomedical Engineering 2014; 61:4.

[3] Friganovic K, Kukolja D, Jovic A, Cifrek M, Krstacic G. Optimizing the Detection of Characteristic Waves in ECG Based on Processing Methods Combinations. IEEE Access 2018; 6:50609-50626.

[4] Gümüş B, Yazgı S. Yapay sinir ağı kullanılarak elektrokardiyogram işaretlerinde otomatik kardiyakaritmi tespiti. Elektrik-Elektronik-Bilgisayar ve Biyomedikal Mühendisliği 13. Ulusal kongresi 2009.

[5] Jindal B, Saudagar, Devi ER. Matlab Based GUI for ECG Arrhythmia Detection Using PanTompkin Algorithm. Fifth International Conference on Parallel, Distributed and Grid Computing (PDGC), 2018; 754-759.

[6] Diker A, Avci E, Gedikpinar M. Determination of R-peaks in ECG signal using Hilbert Transform and Pan-Tompkins Algorithms. 25th Signal Processing and Communications Applications Conference (SIU), 2017; 1-4.

[7] Darweesh M, Habte T, Saleh H, Mohammad B, Ismail M. Novel logarithmic ECG feature extraction algorithm based on pan and tompkins. IEEE 59th International Midwest Symposium on Circuits and Systems (MWSCAS), 2016; 1-4.

[8] Mishra A, Mohapatro M. An IoT framework for Bio-medical sensor data acquisition and machine learning for early detection. International Journal of Advanced Technology and Engineering Exploration 2019; 6:112-125.

[9] Devi R, Kalaivani V. Machine learning and IoT-based cardiac arrhythmia diagnosis using statistical and dynamic features of ECG. The Journal of Supercomputing 2020; 76. 10.1007/s11227-019-02873-y.

[10] IoT ECG Monitoring with AD8232 ECG Sensor \& ESP8266. https://how2electronics.com/iot-ecg-monitoring-ad8232-ecg-sensor-esp8266/. Last accessed date 14.08.2021. 
[11] AD8232 Heart Rate Monitor Hookup Guide. https://learn.sparkfun.com/tutorials/ad8232heart-rate-monitor-hookup-guide/connecting-the-hardware. Last accessed date 13.08.2021.

[12] Yang H, Kan C, Liu G, Chen Y. Spatiotemporal Differentiation of Myocardial Infarctions. Automation Science and Engineering, IEEE Transactions on 2013; 10: 938-947.

[13] Kohler B, Hennig C, Orglmeister R. The principles of software QRS detection. IEEE Engineering in Medicine and Biology Magazine 2002; 21: 42-57.

[14] Yeh YC, Wang WJ. QRS complexes detection for ECG signal: The Difference Operation Method. Computer Methods and Programs in Biomedicine 2008; 91:245-254.

[15] Pan J, Tompkins WJ. A Real-Time QRS Detection Algorithm. IEEE Transactions on Biomedical Engineering 1985; 32:230-236.

[16] Hamilton PS, Tompkins WJ. Quantitative Investigation of QRS Detection Rules Using the MIT/BIH Arrhythmia Database. IEEE Transactions on Biomedical Engineering 1986; 33:1157-1165.

[17] Benitez D, Gaydecki PA, Zaidi A, Fitzpatrick AP.The use of the Hilbert transform in ECG signal analysis,Computers in Biology and Medicine 2001; 31:399-406. 